\title{
Infrapatellar Saphenous Neuralgia After TKA Can Be Improved With Ultrasound-guided Local Treatments
}

\author{
Steven Clendenen MD, Roy Greengrass MD, \\ Joseph Whalen MD, PhD, Mary I. O'Connor MD
}

Published online: 26 July 2014

(C) The Association of Bone and Joint Surgeons (B) 2014

\begin{abstract}
Background Current opinion suggests that in some patients, chronic pain after total knee arthroplasty (TKA) has a neuropathic origin. Injury to the infrapatellar branch of the saphenous nerve (IPSN) has been implicated as a cause of medial knee pain; however, local treatments for this condition remain controversial.

Questions/purposes We sought to explore the efficacy of local treatment to the IPSN in patients with persistent medial knee pain after TKA.
\end{abstract}

Each author certifies that he or she, or a member of his or her immediate family, has no funding or commercial associations (eg, consultancies, stock ownership, equity interest, patent/licensing arrangements, etc) that might pose a conflict of interest in connection with the submitted article.

All ICMJE Conflict of Interest Forms for authors and Clinical Orthopaedics and Related Research ${ }^{\mathbb{R}}$ editors and board members are on file with the publication and can be viewed on request. Clinical Orthopaedics and Related Research ${ }^{\mathbb{R}}$ neither advocates nor endorses the use of any treatment, drug, or device. Readers are encouraged to always seek additional information, including FDA-approval status, of any drug or device prior to clinical use. Each author certifies that his or her institution approved the human protocol for this investigation, that all investigations were conducted in conformity with ethical principles of research, and that informed consent for participation in the study was obtained.

S. Clendenen $(\bowtie)$, R. Greengrass

Department of Anesthesiology, Mayo Clinic, 4500 San Pablo

Road South, Jacksonville, FL 32224, USA

e-mail: Clendenen.steven@mayo.edu

J. Whalen, M. I. O'Connor

Department of Orthopedic Surgery, Mayo Clinic, Jacksonville, FL, USA
Methods In this retrospective series, 16 consecutive patients with persistent medial knee pain after primary or revision TKA were identified after other potential etiologies of knee pain were excluded. Using advanced ultrasound imaging to identify the IPSN, hydrodissection of the nerve from the adjacent interfascial planes was performed followed by corticosteroid injection (local treatment). In two patients, radiofrequency ablation of the IPSN was subsequently performed for recurrent symptoms. The outcome measure of this study was patient-reported relief of medial knee pain based on a visual analog scale (VAS) score of 0 to 10 either at rest or with activity, whichever resulted in more pain for the patient. Followup was at a minimum of 6 months (median, 9 months; range, 6-12 months). Before the procedure, the median highest VAS pain score, either at rest or with activity, was 8 of 10 (range, 6-10).

Results Local injections to the infrapatellar saphenous nerve (one or two injections) improved medial pain after TKA to a VAS score of 0 or 1 in nine of our 16 patients. Three patients reported pain improvement to VAS levels of 3 to 4 . Of the remaining four patients, two did not have improvement with VAS scores of 8 , and two underwent subsequent radiofrequency ablation of the IPSN with resolution of pain in one patient.

Conclusions In summary, we believe injury to the IPSN may be an underappreciated cause of persistent medial pain after TKA. We report favorable preliminary results with local treatment to the nerve in nine of our 16 patients, suggesting that the neuritis is a reversible process in some patients; however, because of the possibility of a placebo effect, we believe this treatment modality should be tested in a randomized, placebo-controlled trial.

Level of Evidence Level IV, therapeutic study. See Guidelines for Authors for a complete description of levels of evidence. 


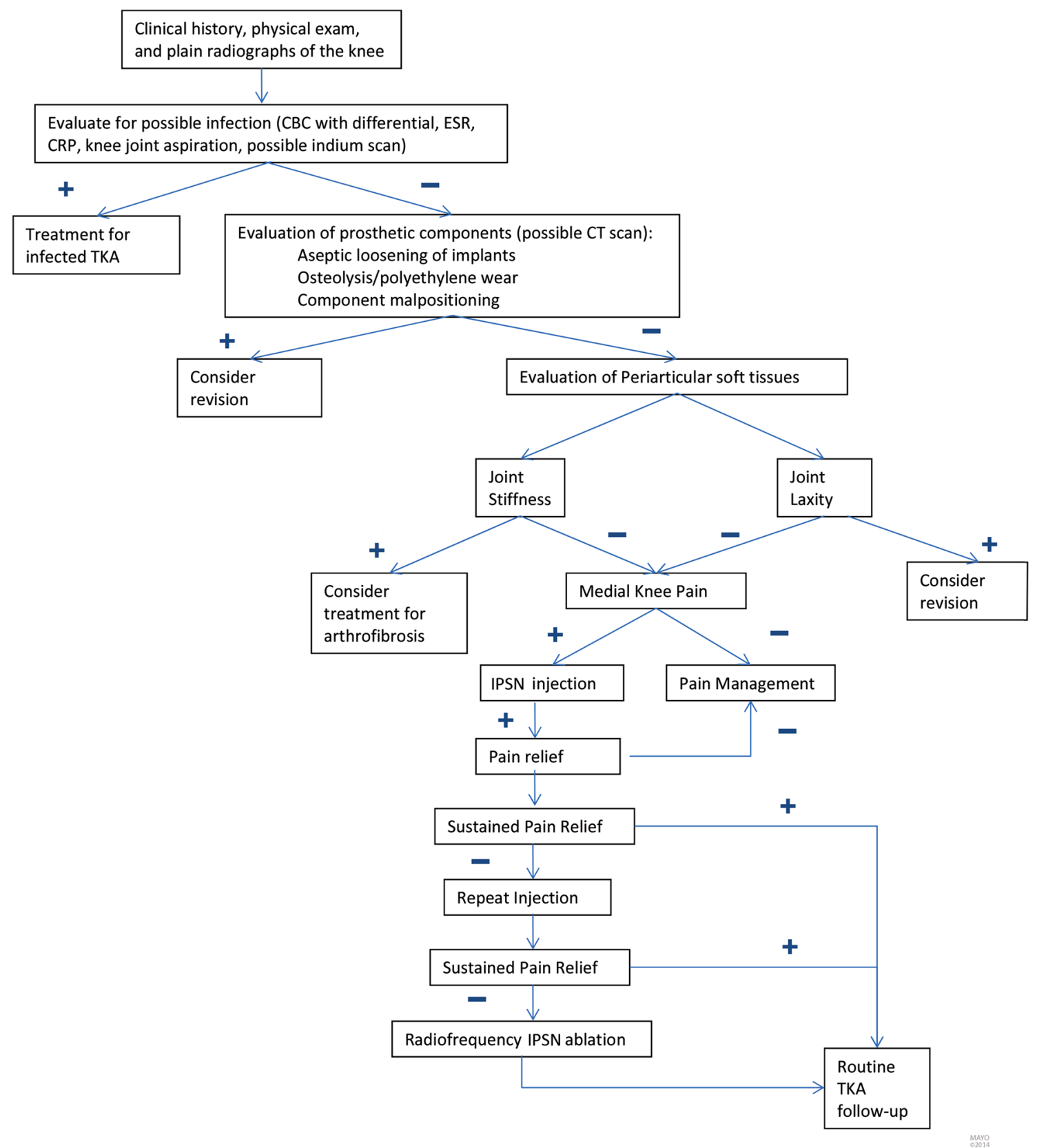

Fig. 1 Algorithm shows evaluation of a patient with a painful TKA. CBC = complete blood count; ESR = erythrocyte sedimentation rate; $\mathrm{CRP}=\mathrm{C}$-reactive protein. Patients must be evaluated for other causes of knee pain before a diagnosis of neuritis of the IFSN is made. By permission of Mayo Foundation for Medical Education and Research. All rights reserved.

\section{Introduction}

Infrapatellar neuroma is a known etiology of persistent knee pain after TKA [8], but injury to the infrapatellar branch of the saphenous nerve (IPSN) may be an underappreciated cause of chronic medial knee pain. The anatomy of the infrapatellar branch of the saphenous nerve makes it uniquely susceptible to injury during knee surgery. The nerve may be most at risk for damage from the medial retractors that are placed during total knee surgery because it exits the sartorial fascia at the distal end of Hunter's canal. With the knee in flexion during the surgical procedure and a retractor that levers out against the medial tibia, the nerve may be under tension and a stretch neurapraxia could result. A thigh tourniquet, commonly used during the surgery to prevent blood loss, may further increase the tension by proximally tethering the nerve. 


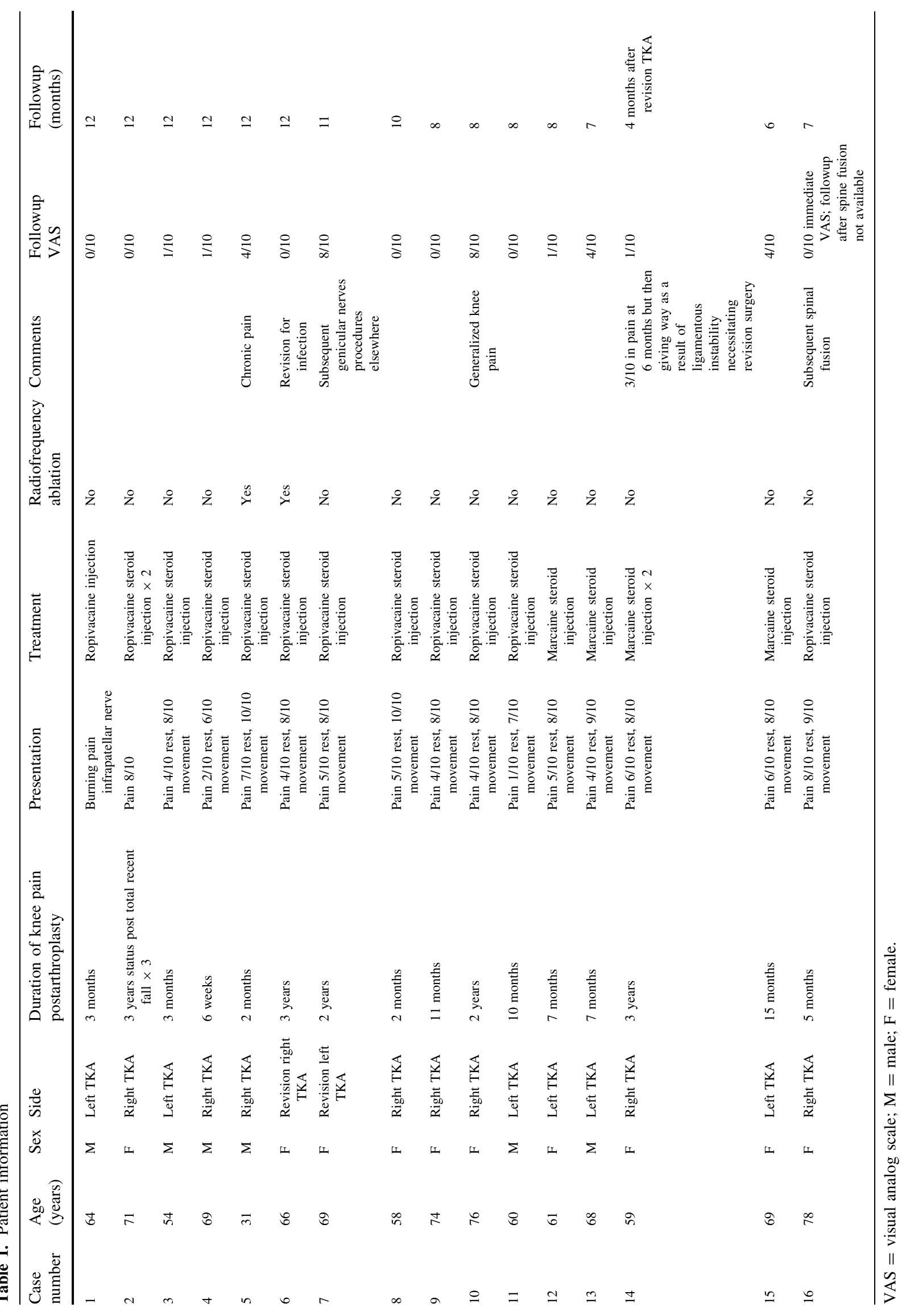




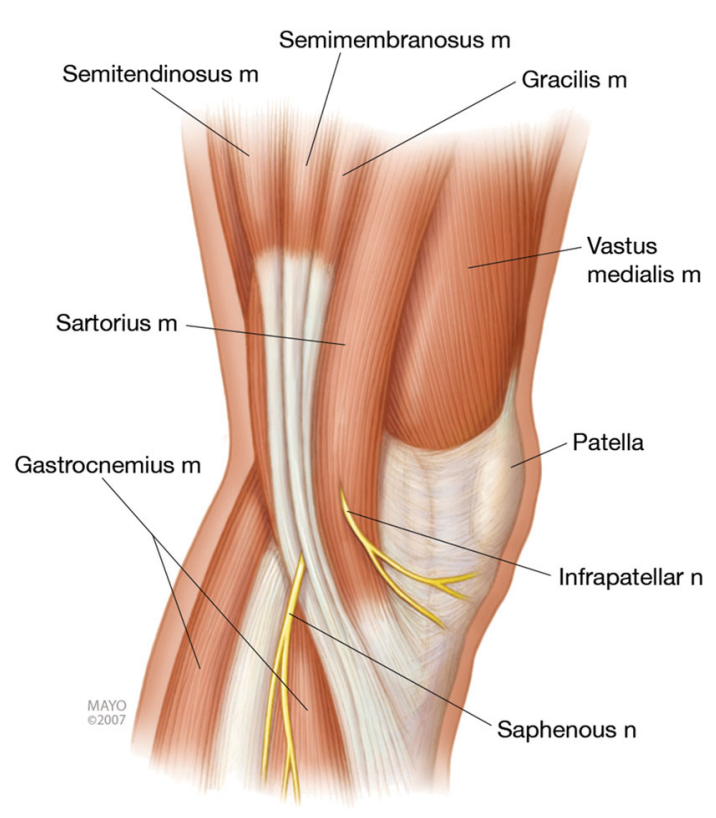

Fig. 2 Anatomy of the IPSN is shown. By permission of Mayo Foundation for Medical Education and Research. All rights reserved.

Adhesions may develop between the injured nerve and adjacent fascial planes, resulting in neuritis.

\section{Rationale}

Challenged by patients with persistent medial knee pain after TKA without a clear etiology for their discomfort, we began to suspect neuritis of the IPSN as a possible etiology and sought to develop and evaluate a treatment protocol for it. The approach involves making a specific diagnosis using advanced ultrasound imaging and nerve stimulation to identify the IPSN followed by local treatment with corticosteroid injection. We are not aware of studies of comparable approaches and present a case series as a proof of concept.

The purpose of this study was to evaluate the efficacy of local corticosteroid injections to the ISPN for reducing ISPN-mediated knee pain after TKA at a minimum followup of 6 months.

\section{Patients and Methods}

\section{Study Design and Setting}

This study is a clinical series of 16 patients evaluated in the Department of Orthopaedic Surgery at the Mayo Clinic, Jacksonville, FL, USA, who were found to have persistent medial knee pain after TKA. None of the patients were lost to followup. After institutional review board approval, a retrospective chart review was performed in 16 such patients who were evaluated between February 2012 and June 2013. Patient-reported results were determined either by clinical visits or by a telephone interview with a minimum of 6 months followup (median, 9 months; range, 6-12 months).

\section{Participants/Study Subjects}

All patients included in this study had persistent medial knee pain after primary or revision TKA and an evaluation, which did not diagnose other etiologies for the pain (Fig. 1). Of the 16 patients, 10 were women and six were men. Two had undergone revision TKA at our institution, including one patient who presented with an infected TKA that had been performed elsewhere (Table 1). All patients had an anterior longitudinal incision.

Patients presented with medial knee pain in the region of the IPSN (Fig. 2) of varying duration (6 weeks to 3 years). They reported that their discomfort ranged from "aching" to "sharp and burning." There was no absolute consistent descriptor for the pain in this small group of patients. Furthermore, the location of the pain could vary with discomfort in some patients radiating around the anterior aspect of the knee toward the lateral compartment or distally into the proximal medial lower leg. The consistent clinical symptom was pain in the medial knee region. Patients were evaluated using a visual analog pain scale (VAS) $(0=$ no pain, $10=$ worst imaginable pain). On clinical examination, patients had tenderness to palpation of this region. A Tinel's test to the medial knee region did not typically result in radicular symptoms but did produce discomfort in the medial knee region. Quadriceps function was intact with all patients having strength to resistance of knee extension. Before the injections of the ISPN, VAS pain scores were recorded for 15 of 16 patients and VAS scores at rest or with movement detailed were recorded in 14 of these 15 patients (Table 1). VAS scores were higher with movement. Of the 15 patients with baseline VAS scores, the highest median VAS pain score was 8 of 10 (range, 6-10).

\section{Description of Experiment, Treatment, or Surgery}

Patients were evaluated and treated by an anesthesiologist (SC) with experience in the use of ultrasound imaging of peripheral nerves. The maximum point of tenderness to palpation along the course of the IPSN was identified and marked. Using a linear array $12-4-\mathrm{MHz}$ transducer and portable ultrasound machine (Sparq; Philips Medical, Andover, MA, USA), the IPSN was identified traversing between the superficial fascia and sartorius muscle. An 


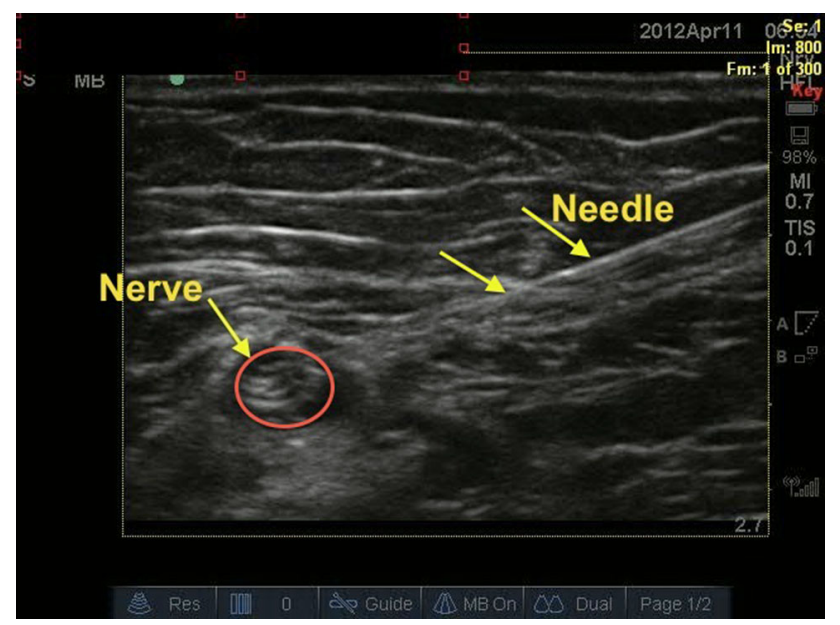

Fig. 3 A 22-G stimulating needle was advanced to the nerve to confirm the infrapatellar branch of the saphenous nerve.

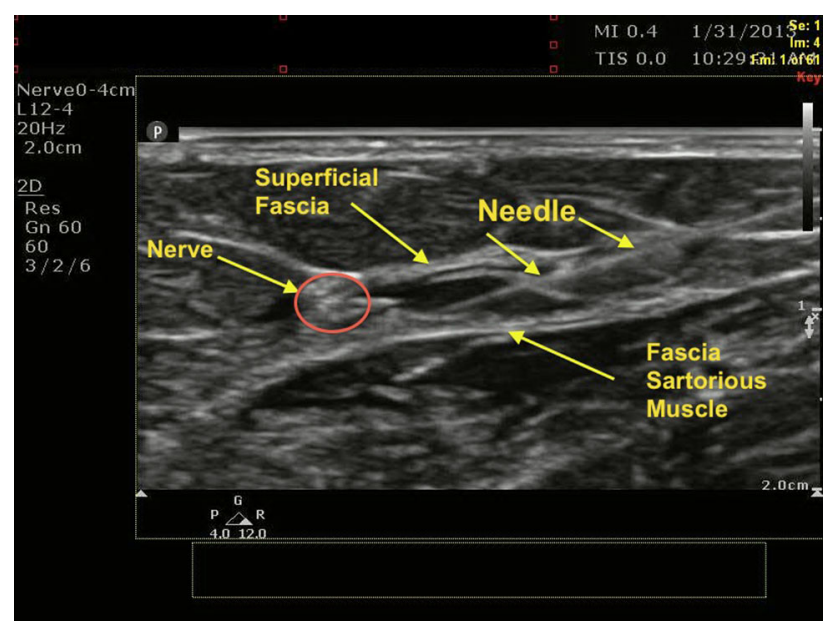

Fig. 4 A mixture of local anesthesia and steroids was injected to hydrodissect the fascial planes and "free up" the infrapatellar branch of the saphenous nerve.

insulated 22-g $\times$ 2-inch block needle (Stimuplex; B Braun, Melsungen, Germany) was advanced with stimulator settings of $2 \mathrm{~Hz}, 0.5 \mathrm{~mA}$, and pulse duration set at $1 \mathrm{~ms}$ (Stimuplex HNS 12; B Braun). The location of the nerve was confirmed by eliciting a paresthesia with electrical stimulation (Fig. 3). A fluid mixture of $20 \mathrm{mg}$ methylprednisolone and $5 \mathrm{~mL} 1.0 \%$ ropivacaine (Fig. 1) or $0.5 \%$ Marcaine was prepared. With an optimal cross-sectional view of the nerve, the 22-g needle was advanced adjacent to the nerve, and the fluid mixture was injected to separate or hydrodissect the fascial planes and "free up" the infrapatellar nerve, which may have adhered or scarred to the fascia (Fig. 4). In our first patient, only ropivacaine was injected.

Patients who did not respond to hydrodissection and corticosteroid injection or had recurrent pain after single or repeated injections were considered for radiofrequency ablation of the IPSN using a 5-cm long, 5-mm active-tip radiofrequency denervation needle (Baylis Medical Company, Montreal, Quebec, Canada) under ultrasound guidance. Two to four ablations were carried out at $80^{\circ} \mathrm{C}$ for 90 seconds using a radiofrequency generator (Baylis Pain Management Generator; Baylis Medical Company). After the lesions, $4 \mathrm{mg}$ dexamethasone was injected before removing the needle.

Indications for repeat injection included a temporary good response to the initial injection. Indications for radiofrequency ablation (RFA), which was used for patients whose pain persisted despite corticosteroid treatment, included significant temporary pain reduction following the injection that could be reproduced by ablating the nerve at that same site.

\section{Aftercare}

After any procedure performed in this study, patients were allowed to return to their preprocedure level of activity.

\section{Description of Followup Routine}

Patients were either seen for clinical followup or contacted by telephone to ensure a minimum of 6 months followup after the last treatment was available for study analysis.

\section{Variables, Outcome Measures, Data Sources, and Bias}

The outcome measure of this study was patient-reported relief of medial knee pain using the VAS. Patients were asked to report their typical maximum level of knee pain, either at rest or with movement, and this was recorded as their level of knee pain. VAS scores were not specifically identified for when the patient was at rest versus active in this retrospective study. Variables studied included the number and effectiveness of local procedures to achieve pain improvement.

No patients were lost to followup.

\section{Statistical Analysis and Study Size}

As a result of the small number of patients in this study, no statistical analysis was performed.

\section{Results}

Local treatment with hydrodissection of the IPSN from the adjacent interfascial planes followed by corticosteroid injection was effective in reducing persistent medial knee 
pain after TKA in nine of our 16 patients (VAS score of 0 or 1) with one (eight patients) or two (one patient) procedures. Three patients had partial improvement with VAS scores of 3 or 4 at followup (Table 1). Of the remaining four patients, two did not have improvement with VAS scores of 8 , and two underwent subsequent RFA of the IPSN with resolution of pain in one patient.

\section{Discussion}

\section{Background and Rationale}

Persistent knee pain after TKA continues to be a challenging problem for patients and physicians. Despite an extensive clinical evaluation, the etiology of pain may be elusive. For patients with pain predominantly in the medial knee region, neuritis of the IPSN may be the cause of their persistent pain. In a study of 113 TKAs, $60 \%$ of patients had subjective numbness in their knee region and $4.5 \%$ had significant problems with hypersensitivity that interfered with activities of daily living [6], which are findings that support injury to the IPSN as a factor in these symptoms.

Variable results have been published regarding treatment of neuritis of the IPSN. Romanoff et al. [11] reported $80 \%$ of 30 patients improved with local injection with 11 of 27 patients with knee pain having had prior knee arthroscopy. However, Worth et al. [12] reported that in 14 patients pain improvement was not sustained after local injection in any patient, and most had some type of prior knee surgery. Surgical treatment with excision of the saphenous nerve may be more predictable than local injection therapy with positive results in over $80 \%$ of patients in three series $[2,3,10]$.

To our knowledge, there have been no clinical series or comparative trials reported that discuss a technique similar to that used in this series. We therefore sought to develop a specific screening and treatment protocol and investigated the effect of hydrodissection of the IPSN from adjacent facial planes in combination with corticosteroid injection as a treatment for such pain and reported our initial series of 16 patients. With accurate identification of the IPSN through advanced ultrasound imaging techniques, we found this technique to be effective in reducing medial knee pain in nine of 16 patients with one (eight patients) or two (one patient) procedures. Whether this less invasive ultrasound-guided technique can be comparable in effectiveness to surgical excision of the nerve will require more clinical study.

\section{Limitations}

This study has a number of limitations. It is a small retrospective study of only 16 patients with the potential for selection bias. We do not know the total number of patients who had undergone TKA seen in our department with persistent medial knee pain or whether some patients were offered an evaluation for potential injection and declined. This study does not identify the incidence of neuritis of the IPSN after TKA, which is also an important question. Furthermore, this study does not identify if neuritis of the IPSN was present before TKA nor does it clearly identify specific causative factors during the surgery that could have resulted in injury to the IPSN. Finally, our followup is short, and pain may have recurred in some patients who responded that their discomfort was resolved. We believe our findings are important to promoting greater exploration of this important clinical problem. Finally, and most importantly, in a small series of patients whose treatment was not blinded and consisted of a focused intervention evaluated at short term, the placebo effect cannot be excluded as a possible explanation. The proportion of our patients reporting success with treatment (nine of 16) also leaves open this possibility; placebocontrolled surgical trials, although rare, have documented patient improvement with sham procedures [1, 4, 5, 9]. A randomized, blinded, placebo-controlled trial would be the best research protocol to evaluate the effectiveness of local treatment to the IPSN, although we do not believe that at our center we could effectively enroll patients in such a study.

We are unaware of prior studies treating neuritis of the IPSN with a similar protocol. We did identify a study in which RFA was used to treat pain in patients with refractory anteromedial pain that was secondary to osteoarthritis who were also considered at high risk for TKA. Postablation patients reported a significant decrease in knee pain measured by VAS and WOMAC pain subscales, but as anticipated, there was little improvement in WOMAC stiffness and physical function subscales. This simple and effective treatment should be considered to improve the quality of life in patients who are considered an unacceptable risk to undergo TKA [7].

\section{Conclusions}

We are encouraged by our preliminary results, which suggest that hydrodissection of fascial planes adjacent to the IPSN in combination with a corticosteroid injection is effective for some patients as a single or repeat procedure. Our experience with RFA is very limited, and conclusions cannot be drawn regarding its effectiveness. However, because of the possibility of a placebo effect, we suggest this treatment modality be tested further in a randomized, placebo-controlled trial. 
Acknowledgments We thank Ms. Kelly Viola ELS, for her assistance in manuscript preparation.

\section{References}

1. Cobb LA, Thomas GI, Dillard DH, Merendino KA, Bruce RA. An evaluation of internal-mammary-artery ligation by a doubleblind technic. N Engl J Med. 1959;260:1115-1118.

2. Dellon AL, Mont MA, Mullick T, Hungerford DS. Partial denervation for persistent neuroma pain around the knee. Clin Orthop Relat Res. 1996;329:216-222.

3. Ducic I, Levin M, Larson EE, Al-Attar A. Management of chronic leg and knee pain following surgery or trauma related to saphenous nerve and knee neuromata. Ann Plast Surg. 2010;64:35-40.

4. Freed CR, Greene PE, Breeze RE, Tsai WY, DuMouchel W, Kao R, Dillon S, Winfield H, Culver S, Trojanowski JQ, Eidelberg D, Fahn S. Transplantation of embryonic dopamine neurons for severe Parkinson's disease. N Engl J Med. 2001;344:710-719.

5. Guyuron B, Reed D, Kriegler JS, Davis J, Pashmini N, Amini S. A placebo-controlled surgical trial of the treatment of migraine headaches. Plast Reconstr Surg. 2009;124:461-468.
6. Hopton BP, Tommichan MC, Howell FR. Reducing lateral skin flap numbness after total knee arthroplasty. Knee. 2004;11:289291.

7. Ikeuchi M, Ushida T, Izumi M, Tani T. Percutaneous radiofrequency treatment for refractory anteromedial pain of osteoarthritic knees. Pain Med. 2011;12:546-551.

8. Kachar SM, Williams KM, Finn HA. Neuroma of the infrapatellar branch of the saphenous nerve a cause of reversible knee stiffness after total knee arthroplasty. J Arthroplasty. 2008;23: 927-930.

9. Moseley JB, O’Malley K, Petersen NJ, Menke TJ, Brody BA, Kuykendall DH, Hollingsworth JC, Ashton CM, Wray NP. A controlled trial of arthroscopic surgery for osteoarthritis of the knee. N Engl J Med. 2002;347:81-88.

10. Nahabedian MY, Johnson CA. Operative management of neuromatous knee pain: patient selection and outcome. Ann Plast Surg. 2001;46:15-22.

11. Romanoff ME, Cory PC, Kalenak A, Keyser GC, Marshall WK. Saphenous nerve entrapment at the adductor canal. Am J Sports Med. 1989;17:478-481.

12. Worth RM, Kettlekamp DB, Defalque RJ, Underwood DK. Saphenous nerve entrapment: a cause of medial knee pain. Am J Sports Med. 1984;12:80-81. 